\title{
Florística, estrutura e formas de vida do estrato inferior de uma Floresta Ombrófila Densa Aluvial, Pará, Brasil
}

\author{
Floristic, structure and life forms of lower stratum in a Alluvial Dense Rain Forest, \\ Pará state, Brazil
}

Elayne Oliveira Braga ${ }^{\mathrm{I}}$, Mário Augusto Gonçalves Jardim ${ }^{\mathrm{II}}$

Resumo

Este estudo teve como objetivo descrever a composição florística, a estrutura e as formas de vida do estrato inferior de uma floresta ombrófila densa aluvial na Área de Proteção Ambiental Ilha do Combu, Belém, Pará. Foram selecionadas quatro parcelas de $20 \mathrm{~m}$ x $20 \mathrm{~m}$ (0,16 ha) divididas em 1.289 subparcelas de $1 \mathrm{~m}^{2}$. Foi classificada a categoria de tamanho e a forma de vida das espécies. Calculou-se a densidade, frequência, categoria de tamanho e regeneração natural relativa. A diferença de abundância, riqueza, índice de Shannon e equabilidade entre as categorias foi testada com ANOVA. Foram amostradas 33 famílias, 63 gêneros, 79 espécies e 26.885 indivíduos. Fabaceae, Araceae, Arecaceae, Acanthaceae e Meliaceae apresentaram maior riqueza. O Índice de Shannon e Equabilidade foram de 2,63 e 0,60, respectivamente para a área total. Pariana campestris e Anthurium sinuatum apresentaram maior densidade, categoria de tamanho e regeneração natural relativa. As herbáceas apresentaram maior porcentagem de indivíduos e regeneração natural relativa e as árvores a maior riqueza. CT1 $(15 \mathrm{~cm} \geq \mathrm{AT})$ foi diferente das demais categorias, apresentando menor abundância e riqueza. Conclui-se que a dominância de herbáceas em porcentagem de indivíduos e regeneração natural relativa e a menor abundância e riqueza na CT1 é consequência das atividades antrópicas realizadas como o manejo intensivo da palmeira-açaí (Euterpe oleracea Mart.) nas proximidades das áreas inventariadas.

Palavras-chave: Floresta de várzea, Inventário florístico; Estuário amazônico

\begin{abstract}
The study aimed to describe the floristic composition and structure and life forms to lower stratum in a alluvial dense rain forest in the Environmental Protection Area of Combu Island, Belém, Pará state. Four plots were selected from $20 \mathrm{~m}$ x $20 \mathrm{~m}$ (0.16 ha) divided into 1.289 subplots of $1 \mathrm{~m}^{2}$. The size category and the life forms of the species were classified. Density, frequency, size category and natural regeneration relative were calculated. The difference in abundance, richness, Shannon index and evenness between categories was tested using ANOVA. All 33 families were found, 63 genera, 79 species and 26,885 individuals. Fabaceae, Araceae, Arecaceae, Acanthaceae and Meliaceae had higher richness. The Shannon index and Evenness were 2.63 and 0.60, respectively for the total area. Pariana campestris and Anthurium sinuatum showed higher density, size category and natural regeneration relative. Herb showed the highest percentage of individuals and natural relative regeneration and tree richest. CT1 $(15 \mathrm{~cm} \geq \mathrm{AT})$ was different from the other categories, with lower abundance and richness. It is concluded that the herb dominance in percentage of individuals and natural relative regeneration and the lower abundance and richness in CT1 is a consequence of the anthropic activities carried out such as the intensive management of açaí palm (Euterpe oleracea Mart.) In the vicinity of the inventoried areas.
\end{abstract}

Keywords: Floodplain forest; Floristic inventory; Amazon estuary

Engenheira Florestal, MSc., Pesquisador Autônomo, Passagem Napoleão Laureano, 1936, CEP 66073-640, Belém (PA), Brasil. elaynnebraga@ hotmail.com (ORCID: 0000-0001-9033-5602)

II Engenheiro Florestal, PhD., Pesquisador Titular da Coordenação de Botânica, Museu Paraense Emilio Goeldi, Museu Paraense Emílio Goeldi, Av.Magalhães Barata, 376, CP 399, CEP 66040-170, Belém (PA), Brasil. jardim@museu-goeldi.br (ORCID: 0000-0003-1575-1248) 


\section{Introdução}

A avaliação da composição e da estrutura de uma comunidade vegetal é importante para o entendimento do ecossistema e, consequentemente, para garantir o seu uso sustentável (MENDES et al., 2013). Em florestas tropicais, a ocorrência das espécies que determinarão a composição florística dependerá de um conjunto de fatores, entre eles o banco de sementes, o fluxo e a dispersão de sementes, a intensidade luminosa e o tipo de solo (COELHO et al., 2003). $\mathrm{Na}$ floresta ombrófila densa aluvial da região amazônica, conhecida como "floresta de várzea", a dinâmica da inundação é um fator ambiental que contribui na composição, na estrutura e no padrão de distribuição das plantas regenerantes (WITTMANN; JUNK, 2003; MARINHO et al., 2013).

Especificamente nas várzeas do estuário amazônico, por conta da sua histórica importância ecológica e econômica, estudos florísticos têm sido bem documentados, contudo, a maioria se limita a levantamentos da composição e dos aspectos estruturais de plantas com diâmetro igual ou superior a $10 \mathrm{~cm}$ e acima de um metro de altura (BATISTA et al., 2011; LAU; JARDIM, 2013; SANTOS et al., 2014). Poucos estudos levaram em consideração plantas com altura menor que 1 m (BATISTA; JARDIM, 2013) e as diferentes formas de vida do estrato inferior, como árvores, arbustos, ervas, lianas, epífitas e hemiepífitas (MAUÉS et al., 2011).

As plantas do estrato inferior são responsáveis por promover a estabilidade e a continuidade das populações vegetais, porém, a permanência de uma espécie está diretamente relacionada com a sua representatividade em número de indivíduos e distribuição em altura (VIANA; JARDIM, 2013).

Em uma abordagem ecológica, as espécies do estrato inferior formam um nicho ecológico importante para o estabelecimento das espécies que também poderão constituir os demais estratos (OLIVEIRA; AMARAL, 2005). Concomitantemente, servem de alimento para a fauna local, retêm sedimentos e protegem as camadas superficiais do solo contra a erosão (PIEDADE; SCHOENGART; JUNK, 2005).

O levantamento qualitativo e quantitativo do estrato inferior permite reconhecer a função das espécies na comunidade, bem como suas preferências em habitat e as relações ecológicas inter e intraespecíficas (APARICIO et al., 2014). As informações sobre a composição e a estrutura deste estrato são importantes para o planejamento do manejo e para a aplicação de práticas silviculturais direcionadas ao aproveitamento contínuo da floresta (GAMA et al., 2003).

$\mathrm{Na}$ Área de Proteção Ambiental (APA) Ilha do Combu, a relação entre a floresta de várzea e a comunidade humana é basicamente de subsistência (LAU; JARDIM, 2014), o extrativismo do fruto de Euterpe oleracea Mart, por exemplo, é a base econômica da população local. Diante disso, as informações qualitativas e quantitativas do estrato inferior são valiosas para se conhecer o estoque desta floresta, para prever o desenvolvimento futuro e consequentemente propor medidas de conservação e manutenção das espécies vegetais desse ambiente. O objetivo deste estudo foi descrever a composição florística, a estrutura e as formas de vida do estrato inferior em uma floresta ombrófila densa aluvial na Área de Proteção Ambiental Ilha do Combu, no munícipio de Belém, Pará.

\section{Material e método}

O estudo foi realizado na Área de Proteção Ambiental (APA) Ilha do Combu (00³8'29" $S$ e $47^{\circ} 31^{\prime} 54^{\prime}$ W), com uma área total de 1.500 ha situada à margem esquerda do Rio Guamá a 7 $\mathrm{km}$ em linha reta do Munícipio de Belém, Pará (AMARAL et al., 2012). Na APA, a vegetação é exclusivamente de Floresta Ombrófila Densa Aluvial (IBGE, 2012). A temperatura média anual é de $27^{\circ} \mathrm{C}$ e a precipitação anual de $2.500 \mathrm{~mm}$. O solo predominante é o Glei Pouco Húmico, com alta porcentagem de silte e argila (JARDIM; VIEIRA, 2001).

Na APA Combu foi selecionada uma área, com solo argiloso mal drenado, topografia

Ci. Fl., Santa Maria, v. 29, n. 3, p. 1048-1059, jul./set. 2019 
plana, próxima a afluentes hídricos, sujeita a inundação, pouco densa, com pouca luminosidade no interior da floresta e as proximidades de áreas em que ocorre o manejo da palmeira-açaí por meio de desbastes de estipes e raleamento da floresta (QUARESMA; JARDIM, 2013). Nesta área foram delimitadas quatro parcelas amostrais de $400 \mathrm{~m}^{2}(20 \mathrm{~m} \times 20 \mathrm{~m})$ equidistantes aproximadamente $80 \mathrm{~m}$, perfazendo uma área amostral de 0,16 ha. Parcela $1=\left(01^{\circ} 30^{\prime} 30,57^{\prime \prime} \mathrm{S}\right.$ e $\left.048^{\circ} 27^{\prime} 40,89^{\prime \prime} \mathrm{W}\right)$, Parcela $2\left(01^{\circ} 30^{\prime} 29^{\prime \prime}\right.$ S e $048^{\circ} 27^{\prime} 40,54^{\prime \prime}$ W), Parcela $3\left(01^{\circ} 30^{\prime} 29,86^{\prime \prime}\right.$ S e $048^{\circ} 27^{\prime} 41,32^{\prime \prime}$ W) e Parcela $4\left(01^{\circ} 30^{\prime} 29,49^{\prime \prime}\right.$ S e $\left.048^{\circ} 27^{\prime} 41,95^{\prime \prime} \mathrm{W}\right)$.

No período de dezembro/2014 a julho/2015, foram dispostas 1.289 subparcelas de $1 \mathrm{~m}^{2}$ utilizando-se um quadrado formado com quatro tubos de PVC de $1 \mathrm{~m}$, unidos por conexões de PVC hidráulica, segundo a metodologia adotada por Maués et al. (2011). Para atingir a maior área amostral possível, em cada parcela, a partir de um ponto inicial, o quadrado foi disposto a espaços regulares de $20 \mathrm{~cm}$.

Nas subparcelas foi realizada a identificação e quantificação das espécies com seus respectivos indivíduos com altura total $(\mathrm{AT}) \leq 1 \mathrm{~m}$. Os indivíduos foram classificados em categorias de tamanho $(\mathrm{CT}):(\mathrm{CT} 1=15 \mathrm{~cm} \geq \mathrm{AT} ; \mathrm{CT} 2=15,1 \mathrm{~cm} \leq \mathrm{AT} \leq 30 \mathrm{~cm} ; \mathrm{CT} 3=30,1 \mathrm{~cm} \leq$ AT $\leq 1 \mathrm{~m}$ ), utilizando uma vara graduada, posicionada no centro da subparcela, metodologia adaptada de Maués et al. (2011).

A identificação botânica in loco foi realizada por um parabotânico do Museu Paraense Emilio Goeldi (MPEG). Para as espécies de difícil identificação foi coletado material botânico para comparação com as coleções do Herbário João Murça Pires (Minas Gerais). A organização sistemática das famílias foi feita de acordo com APG III (ANGIOSPERM PHYLOGENY GROUP, 2009) para as angiospermas e Smith et al. (2006) para samambaias. A atualização taxonômica das espécies e de seus autores foi realizada mediante consulta ao banco de dados do Missouri Botanical Garden (MISSOURI BOTANICAL GARDEN, 2015). A classificação das formas de vida das espécies foi efetuada de acordo com Veloso, Rangel Filho e Lima (1991) considerando as seguintes especificações: Arbusto (Arb), Árvore (Arv), Epífita (Epi), Erva (Erv), Hemiepífita (Hem), Liana (Lia) e Palmeira (Pal).

A diversidade de espécies foi avaliada por meio do Índice de Shannon-Wienner ( $\left.\mathrm{H}^{\prime}\right)$ (MAGURRAN, 1988) e Equabilidade (J') (PIELOU, 1977). Para determinar a estrutura horizontal foram estimados os parâmetros absolutos e relativos de frequência e densidade. Para analisar a estrutura vertical, foi determinada a Categoria de Tamanho Relativa por espécie (CT\%), calculada de acordo com Finol (1971):

$$
\mathrm{CT} \%=\frac{\mathrm{CT}_{\mathrm{sp}}}{\Sigma \mathrm{CT}} \times 100
$$

Em que: $\left.\mathrm{CT}_{\mathrm{sp}}=\left[\left(\mathrm{n}_{1} \mathrm{~N}_{1}+\ldots+\mathrm{n}_{3} \mathrm{~N}_{3}\right) \div \mathrm{N} \times 100\right)\right] ; \mathrm{CT}_{\mathrm{sp}}=$ Categoria de tamanho de cada espécie; $\mathrm{n}_{1 . . .3}=$ número de indivíduos de cada espécie, nas categorias de tamanho de 1 a $3 ; \mathrm{N}_{1 . .3}=$ número total de índivíduos nas categorias de tamanho 1 a $3 ; \mathrm{N}$ = número total de indivíduos da amostragem; $\mathrm{CT}$ = somatório da categoria de tamanho de todas as espécies.

As espécies foram hierarquizadas em ordem de importância pela Regeneração Natural Relativa ( $\mathrm{Rn} \%)$, obtida pela média dos valores relativos de densidade, frequência e categoria de tamanho (FINOL, 1971).

A Abundância, Riqueza, Equabilidade e Índice de Shannon-Wiener das categorias de tamanho foi comparada com Análise de Variância One-Way (ANOVA) a 5\% de probabilidade, seguido do teste Post Hoc Test de Tukey, sendo a normalidade dos dados determinada pelo teste de Shapiro-Wilk ( $\mathrm{p}>0,05)$. A análise foi realizada no software R 3.2.2 (R CORE TEAM, 2014), por meio do pacote car (FOX et al., 2015). 


\section{Resultados e discussão}

Foram registradas 33 famílias, 63 gêneros, 79 espécies e 26.885 indivíduos. Fabaceae, com 15 espécies, seguida de Araceae e Arecaceae (6) e Acanthaceae e Meliaceae (4) destacaram-se com maior riqueza (Tabela 1). A maior riqueza de Fabaceae nas florestas de várzea do estuário amazônico também foi observada em diversos estudos florísticos que incluíram diferentes formas de vida, apenas a regeneração de árvores (GAMA; BOTELHO; BENTES-GAMA, 2002; GAMA et al., 2003; MAUÉS et al., 2011; BATISTA; JARDIM, 2013) e a vegetação com diâmetro $\geq 10 \mathrm{~cm}$ (ALMEIDA; JARDIM, 2011; BATISTA et al., 2011).

\section{Tabela 1 - Parâmetros fitossociológicos das espécies do estrato inferior amostradas na floresta ombrófila densa aluvial, Área de Proteção Ambiental Ilha do Combu. Listagem organizada por ordem decrescente de $\mathrm{Rn} \%$.}

Table 1 - Phytosociological parameters of species of lower stratum shown in alluvial dense rain forest, Environmental Protection Area of Combu Island. This list was organized by descending order of $\mathrm{Rn} \%$.

\begin{tabular}{|c|c|c|c|c|c|c|c|c|c|c|}
\hline Famílias & Nome Científico & FV & CT1 & CT2 & CT3 & $\mathbf{N}$ & Dr\% & Fr\% & СТ\% & $\mathbf{R n} \%$ \\
\hline Poaceae & Pariana campestris Aubl. & Erv & 41 & 1.948 & 6.094 & 8.083 & 30,07 & 1,94 & 29,60 & 20,53 \\
\hline Araceae & $\begin{array}{c}\text { Anthurium sinuatum Benth. ex } \\
\text { Schott }\end{array}$ & Hem & 333 & 4.854 & 411 & 5.598 & 20,82 & 1,94 & 22,06 & 14,94 \\
\hline Costaceae & Costus arabicus $\mathrm{L}$. & Erv & 42 & 273 & 901 & 1.216 & 4,52 & 1,94 & 4,33 & 3,60 \\
\hline Costaceae & Costus spicatus (Jacq.) Sw. & Erv & 4 & 621 & 542 & 1.167 & 4,34 & 1,46 & 4,52 & 3,44 \\
\hline Myrtaceae & $\begin{array}{c}\text { Syzygium malaccense (L.) Merr. \& } \\
\text { L.M. Perry }\end{array}$ & Arv & 170 & 902 & 185 & 1.257 & 4,68 & 0,49 & 4,52 & 3,23 \\
\hline Fabaceae & Inga laurina (Sw.) Willd. & Arv & 212 & 691 & 71 & 974 & 3,62 & 1,94 & 3,25 & 2,94 \\
\hline Acanthaceae & $\begin{array}{c}\text { Trichanthera gigantea (Bonpl.) } \\
\text { Nees }\end{array}$ & Arv & 85 & 488 & 268 & 841 & 3,13 & 1,94 & 3,03 & 2,70 \\
\hline Piperaceae & Piper hispidum Sw. & Arb & 20 & 468 & 208 & 696 & 2,59 & 1,94 & 2,71 & 2,41 \\
\hline Heliconiaceae & Heliconia psittacorum L. f. & Erv & 85 & 338 & 224 & 647 & 2,41 & 1,94 & 2,25 & 2,20 \\
\hline Fabaceae & $\begin{array}{l}\text { Pterocarpus santalinoides L'Hér. } \\
\text { ex DC. }\end{array}$ & Arv & 48 & 281 & 276 & 605 & 2,25 & 1,94 & 2,17 & 2,12 \\
\hline Fabaceae & Inga edulis Mart. & Arv & 21 & 425 & 113 & 559 & 2,08 & 1,94 & 2,20 & 2,07 \\
\hline Arecaceae & Euterpe oleracea Mart. & $\mathrm{Pal}$ & 10 & 312 & 218 & 540 & 2,01 & 1,94 & 2,08 & 2,01 \\
\hline Marantaceae & $\begin{array}{c}\text { Ischnosiphon obliquus (Rudge) } \\
\text { Körn. }\end{array}$ & Erv & 7 & 255 & 221 & 483 & 1,80 & 1,94 & 1,85 & 1,86 \\
\hline Bignoniaceae & $\begin{array}{c}\text { Memora magnifica (Mart. ex DC.) } \\
\text { Bureau }\end{array}$ & Lia & 5 & 226 & 167 & 398 & 1,48 & 1,94 & 1,54 & 1,65 \\
\hline Araceae & $\begin{array}{l}\text { Dieffenbachia seguine (Jacq.) } \\
\text { Schott }\end{array}$ & Erv & 69 & 165 & 121 & 355 & 1,32 & 1,94 & 1,15 & 1,47 \\
\hline Clusiaceae & Symphonia globulifera L. f. & Arv & 7 & 80 & 223 & 310 & 1,15 & 1,94 & 1,12 & 1,41 \\
\hline Arecaceae & $\begin{array}{c}\text { Socratea exorrhiza (Mart.) H. } \\
\text { Wendl. }\end{array}$ & $\mathrm{Pal}$ & 12 & 190 & 95 & 297 & 1,10 & 1,94 & 1,14 & 1,40 \\
\hline Piperaceae & $\begin{array}{l}\text { Piper hostmannianum (Miq.) C. } \\
\text { DC. }\end{array}$ & Arb & 4 & 120 & 141 & 265 & 0,99 & 1,94 & 1,00 & 1,31 \\
\hline Araceae & $\begin{array}{c}\text { Anthurium pentaphyllum (Aubl.) } \\
\text { G. Don }\end{array}$ & $\mathrm{Hem}$ & 53 & 279 & 3 & 335 & 1,25 & 1,46 & 1,21 & 1,30 \\
\hline
\end{tabular}


Tabela 1 - Continuação...

Table 1 - Continuation...

\begin{tabular}{|c|c|c|c|c|c|c|c|c|c|c|}
\hline Famílias & Nome Científico & FV & CT1 & CT2 & CT3 & $\mathbf{N}$ & Dr\% & Fr\% & CT\% & $\mathbf{R n} \%$ \\
\hline Euphorbiaceae & Hura crepitans L. & Arv & 3 & 69 & 188 & 260 & 0,97 & 1,94 & 0,95 & 1,29 \\
\hline Myristicaceae & $\begin{array}{l}\text { Virola surinamensis (Rol. ex Rottb.) } \\
\text { Warb. }\end{array}$ & Arv & 4 & 126 & 100 & 230 & 0,86 & 1,94 & 0,88 & 1,23 \\
\hline Fabaceae & Inga alba (Sw.) Willd. & Arv & 5 & 174 & 27 & 206 & 0,77 & 1,46 & 0,83 & 1,02 \\
\hline Heliconiaceae & Heliconia bihai (L.) L. & Erv & 8 & 69 & 61 & 138 & 0,51 & 1,94 & 0,51 & 0,99 \\
\hline Acanthaceae & Ruellia graecizans Backer & Erv & 14 & 101 & 22 & 137 & 0,51 & 1,94 & 0,51 & 0,99 \\
\hline Acanthaceae & Ruellia cordifolia Vahl & Lia & 7 & 28 & 100 & 135 & 0,50 & 1,94 & 0,47 & 0,97 \\
\hline Meliaceae & Guarea guidonia (L.) Sleumer & Arv & 10 & 67 & 32 & 109 & 0,41 & 1,94 & 0,40 & 0,92 \\
\hline Euphorbiaceae & $\begin{array}{c}\text { Manihot tripartita (Spreng.) Müll. } \\
\text { Arg. }\end{array}$ & Arb & 42 & 51 & 78 & 171 & 0,64 & 1,46 & 0,51 & 0,87 \\
\hline Meliaceae & Carapa guianensis Aubl. & Arv & - & 12 & 75 & 87 & 0,32 & 1,94 & 0,31 & 0,86 \\
\hline Amaryllidaceae & Crinum americanum $\mathrm{L}$. & Erv & 1 & 28 & 22 & 51 & 0,19 & 1,94 & 0,20 & 0,78 \\
\hline Fabaceae & Zygia latifolia (L.) Fawc. \& Rendle & Arb & 2 & 30 & 5 & 37 & 0,14 & 1,94 & 0,14 & 0,74 \\
\hline Fabaceae & $\begin{array}{c}\text { Machaerium leiophyllum (DC.) } \\
\text { Benth. }\end{array}$ & Lia & - & 11 & 25 & 36 & 0,13 & 1,94 & 0,13 & 0,74 \\
\hline Malvaceae & $\begin{array}{l}\text { Herrania mariae (Mart.) Decne. } \\
\text { ex Goudot }\end{array}$ & Arv & 1 & 3 & 30 & 34 & 0,13 & 1,94 & 0,12 & 0,73 \\
\hline Araceae & $\begin{array}{c}\text { Montrichardia linifera (Arruda) } \\
\text { Schott }\end{array}$ & Erv & - & - & 32 & 32 & 0,12 & 1,94 & 0,11 & 0,72 \\
\hline Arecaceae & Desmoncus orthacanthos Mart. & Lia & - & 2 & 20 & 22 & 0,08 & 1,94 & 0,08 & 0,70 \\
\hline Euphorbiaceae & $\begin{array}{l}\text { Hevea brasiliensis (Willd. ex A. } \\
\text { Juss.) Müll. Arg. }\end{array}$ & Arv & - & 12 & 73 & 85 & 0,32 & 1,46 & 0,31 & 0,69 \\
\hline Burseraceae & Protium krukoffii Swart & Arv & 1 & 33 & 42 & 76 & 0,28 & 1,46 & 0,29 & 0,68 \\
\hline Fabaceae & $\begin{array}{c}\text { Pentaclethra macroloba (Willd.) } \\
\text { Kuntze }\end{array}$ & Arv & - & 15 & 22 & 37 & 0,14 & 1,46 & 0,14 & 0,58 \\
\hline Commelinaceae & Dichorisandra affinis Mart. & Erv & 2 & 8 & 23 & 33 & 0,12 & 1,46 & 0,12 & 0,56 \\
\hline Sapotaceae & $\begin{array}{c}\text { Sarcaulus brasiliensis (A. DC.) } \\
\text { Eyma }\end{array}$ & Arv & - & 20 & 3 & 23 & 0,09 & 1,46 & 0,09 & 0,55 \\
\hline Lecythidaceae & $\begin{array}{c}\text { Eschweilera coriacea (DC.) S.A. } \\
\text { Mori }\end{array}$ & Arv & 1 & 2 & 13 & 16 & 0,06 & 1,46 & 0,05 & 0,52 \\
\hline Hernandiaceae & Hernandia guianensis Aubl. & Arv & - & - & 4 & 4 & 0,01 & 1,46 & 0,01 & 0,50 \\
\hline Fabaceae & $\begin{array}{c}\text { Machaerium ferox (Mart. ex } \\
\text { Benth.) Ducke }\end{array}$ & Lia & 1 & 12 & 18 & 31 & 0,12 & 0,97 & 0,11 & 0,40 \\
\hline Aspleniaceae & Asplenium serratum L. & Epi & 4 & 13 & 14 & 31 & 0,12 & 0,97 & 0,11 & 0,40 \\
\hline Bignoniaceae & $\begin{array}{c}\text { Memora flavida (DC.) Bureau \& } \\
\text { K. Schum. }\end{array}$ & Lia & - & 22 & 6 & 28 & 0,10 & 0,97 & 0,11 & 0,40 \\
\hline Fabaceae & Macrolobium bifolium (Aubl.) Pers. & Arv & 4 & 12 & 2 & 18 & 0,07 & 0,97 & 0,06 & 0,37 \\
\hline Malvaceae & Theobroma cacao L. & Arv & - & 3 & 11 & 14 & 0,05 & 0,97 & 0,05 & 0,36 \\
\hline Moraceae & Ficus maxima Mill. & Arv & - & 7 & 4 & 11 & 0,04 & 0,97 & 0,04 & 0,35 \\
\hline Arecaceae & Desmoncus mitis Mart. & Lia & - & 2 & 9 & 11 & 0,04 & 0,97 & 0,04 & 0,35 \\
\hline Chrysobalanaceae & Parinari excelsa Sabine & Arv & - & 7 & 1 & 8 & 0,03 & 0,97 & 0,03 & 0,34 \\
\hline
\end{tabular}


Tabela 1 - Conclusão...

Table 1 - Conclusion...

\begin{tabular}{|c|c|c|c|c|c|c|c|c|c|c|}
\hline Famílias & Nome Científico & FV & CT1 & CT2 & CT3 & $\mathbf{N}$ & Dr\% & Fr\% & СТ\% & $\mathbf{R n} \%$ \\
\hline Fabaceae & Inga alata Benoist & Arv & 1 & 4 & 3 & 8 & 0,03 & 0,97 & 0,03 & 0,34 \\
\hline Passifloraceae & Passiflora vespertilio $\mathrm{L}$. & Lia & 6 & 2 & 2 & 10 & 0,04 & 0,97 & 0,02 & 0,34 \\
\hline Lomariopsidaceae & Lomariopsis prieuriana Fée & Hem & 1 & 4 & 2 & 7 & 0,03 & 0,97 & 0,02 & 0,34 \\
\hline Araceae & Philodendron acutatum Schott & Hem & - & 6 & - & 6 & 0,02 & 0,97 & 0,03 & 0,34 \\
\hline Malpighiaceae & Hiraea chrysophylla A. Juss. & Lia & - & 1 & 4 & 5 & 0,02 & 0,97 & 0,02 & 0,34 \\
\hline Rubiaceae & $\begin{array}{l}\text { Psychotria colorata (Willd. ex } \\
\text { Roem. \& Schult.) Müll. Arg. }\end{array}$ & Arb & - & - & 4 & 4 & 0,01 & 0,97 & 0,01 & 0,33 \\
\hline Chrysobalanaceae & Licania guianensis (Aubl.) Griseb. & Arv & - & 2 & 1 & 3 & 0,01 & 0,97 & 0,01 & 0,33 \\
\hline Meliaceae & Trichilia micrantha Benth. & Arv & - & 1 & 2 & 3 & 0,01 & 0,97 & 0,01 & 0,33 \\
\hline Araceae & $\begin{array}{l}\text { Urospatha sagittifolia (Rudge) } \\
\text { Schott }\end{array}$ & Erv & - & 1 & 2 & 3 & 0,01 & 0,97 & 0,01 & 0,33 \\
\hline Meliaceae & Trichilia quadrijuga Kunth & Arv & 1 & 1 & 1 & 3 & 0,01 & 0,97 & 0,01 & 0,33 \\
\hline Arecaceae & Astrocaryum murumuru Mart. & Pal & - & 34 & - & 34 & 0,13 & 0,49 & 0,14 & 0,25 \\
\hline Fabaceae & $\begin{array}{l}\text { Macrolobium angustifolium } \\
\text { (Benth.) R.S.Cowan }\end{array}$ & Arv & - & 8 & 8 & 16 & 0,06 & 0,49 & 0,06 & 0,20 \\
\hline Cyperaceae & Scleria gaertneri Raddi & Erv & 7 & 3 & 2 & 12 & 0,04 & 0,49 & 0,02 & 0,18 \\
\hline Fabaceae & Bauhinia guianensis Aubl & Arb & 2 & 4 & - & 6 & 0,02 & 0,49 & 0,02 & 0,18 \\
\hline Dilleniaceae & $\begin{array}{l}\text { Doliocarpus dentatus (Aubl.) } \\
\text { Standl. }\end{array}$ & Lia & - & 5 & - & 5 & 0,02 & 0,49 & 0,02 & 0,18 \\
\hline Fabaceae & Crudia oblonga Benth. & Arv & 1 & 3 & 1 & 5 & 0,02 & 0,49 & 0,02 & 0,17 \\
\hline Lauraceae & $\begin{array}{c}\text { Mezilaurus mahuba (A. Samp.) van } \\
\text { der Werff }\end{array}$ & Arv & - & 1 & 3 & 4 & 0,01 & 0,49 & 0,01 & 0,17 \\
\hline Acanthaceae & Justicia comata (L.) Lam. & Erv & - & 2 & - & 2 & 0,01 & 0,49 & 0,01 & 0,17 \\
\hline Fabaceae & Crudia bracteata Benth. & Arv & - & 1 & - & 1 & 0,00 & 0,49 & 0,00 & 0,16 \\
\hline Arecaceae & Desmoncus polyacanthos Mart. & Lia & - & 1 & - & 1 & 0,00 & 0,49 & 0,00 & 0,16 \\
\hline Dioscoreaceae & $\begin{array}{c}\text { Dioscorea laxiflora Mart. ex } \\
\text { Griseb. }\end{array}$ & Lia & - & 1 & - & 1 & 0,00 & 0,49 & 0,00 & 0,16 \\
\hline Myrtaceae & Eugenia omissa McVaugh & Arv & - & 1 & - & 1 & 0,00 & 0,49 & 0,00 & 0,16 \\
\hline Moraceae & Ficus insipida Willd. & Arv & - & 1 & - & 1 & 0,00 & 0,49 & 0,00 & 0,16 \\
\hline Fabaceae & $\begin{array}{c}\text { Senna quinquangulata (Rich.) H.S. } \\
\text { Irwin \& Barneby }\end{array}$ & Arb & - & 1 & - & 1 & 0,00 & 0,49 & 0,00 & 0,16 \\
\hline Rubiaceae & $\begin{array}{l}\text { Uncaria guianensis (Aubl.) J.F. } \\
\text { Gmel. }\end{array}$ & Lia & - & 1 & - & 1 & 0,00 & 0,49 & 0,00 & 0,16 \\
\hline Rubiaceae & Genipa americana L. & Arv & - & - & 1 & 1 & 0,00 & 0,49 & 0,00 & 0,16 \\
\hline Bignoniaceae & $\begin{array}{l}\text { Manaosella cordifolia (DC.) A.H. } \\
\text { Gentry }\end{array}$ & Lia & - & - & 1 & 1 & 0,00 & 0,49 & 0,00 & 0,16 \\
\hline Malvaceae & Matisia paraensis Huber & Arv & - & - & 1 & 1 & 0,00 & 0,49 & 0,00 & 0,16 \\
\hline Myrtaceae & Myrcia fallax (Rich.) DC. & Arv & - & - & 1 & 1 & 0,00 & 0,49 & 0,00 & 0,16 \\
\hline Clusiaceae & $\begin{array}{c}\text { Rheedia macrophylla (Mart.) } \\
\text { Planch. \& Triana }\end{array}$ & Arv & - & - & 1 & 1 & 0,00 & 0,49 & 0,00 & 0,16 \\
\hline
\end{tabular}

Em que: FV = Forma de vida; CT1, CT2 e CT3 = Categorias de tamanho; $\mathrm{N}$ = Número de indivíduos; Dr\% = Densidade relativa; $\mathrm{Fr} \%$ = Frequência relativa; $\mathrm{CT} \%$ = Categoria de Tamanho relativa; $\mathrm{Rn} \%$ = Regeneração Natural relativa. 
Os valores de Índice de Shannon e Equabilidade para a área total foram de 2,63 e 0,60, respectivamente. É comum encontrar baixos valores de diversidade na floresta ombrófila densa aluvial, que em geral variam entre 1,62 e 3,52 para o Índice de Shannon (ALMEIDA; AMARAL; SILVA, 2004) e de 0,63 a 0,73 para a Equabilidade (SANTOS; JARDIM, 2006; CARIM; JARDIM; MEDEIROS, 2008). A baixa diversidade está associada às restritivas condições ambientais, como a saturação hídrica do solo, que leva a formação de uma comunidade desuniforme, com a dominância de poucas espécies, conhecidas como oligoespécies (ALMEIDA; AMARAL; SILVA, 2004; CARVALHO et al., 2009).

Apenas 31 espécies apresentaram o número de indivíduos maior do que 50, totalizando 26.311 indivíduos, o que correspondeu a 97,86\% daqueles registados. Pariana campestris Aubl, Anthurium sinuatum Benth. ex Schott, Syzygium malaccense (L.) Merr. \& L. M. Perry, Costus arabicus L., Costus spicatus (Jacq.) Sw. e Inga laurina (Sw.) Willd. foram as mais abundantes e responsáveis por $68,04 \%$ do total de indivíduos. Pariana campestris e Anthurium sinuatum, juntas apresentaram maior densidade relativa $(50,89 \%$ do total), categoria de tamanho relativa $(51,66 \%)$ e regeneração natural relativa $(35,47 \%)$. As espécies que apresentaram apenas um indivíduo foram em sua maioria classificadas como árvore (6 espécies) e liana (4 espécies) (Tabela 1).

A concentração de muitos indivíduos em poucas espécies é um padrão comum nas florestas inundáveis (SANTOS; JARDIM, 2006; JARDIM et al., 2013). Entretanto, as espécies com maior número de indivíduos foram em sua maioria ervas com rápido crescimento devido à adoção tanto da reprodução assexuada como da sexuada, neste caso Pariana campestris, Costus arabicus e Costus spicatus (MAUÉS, 2009). Certas espécies produzem muitos propágulos, mas não suportam as inundações diárias e as constantes mudanças nas condições edáficas do solo da várzea, o que pode justificar o baixo número de indivíduos de algumas espécies.

Parina campestris também foi abundante no estrato inferior da floresta de várzea do município de Mazagão, Amapá (RABELO et al., 2000) e em floresta de terra firme na Amazônia Central (OLIVEIRA; AMARAL, 2005). Esta é uma espécie perene, com ampla distribuição no Norte e Oeste da América do Sul e no Brasil (ROCHA; LINS, 2009). Possui características morfológicas adaptativas que a possibilitam colonizar diferentes tipos de ambiente. No interior de florestas densas, cujo fluxo de vento é reduzido, a polinização ocorre principalmente por entomofilia, já em ambientes abertos, a grande produção de pequenos grãos de pólen, associada à alta velocidade do vento, permite a polinização por anemofilia (SODERSTROM; CALDERÓN, 1971).

A dominância de Pariana campestris e Anthurium sinuatum nos parâmetros verticais, CT\% e $\mathrm{Rn} \%$ pode ser resultante da concentração de indivíduos em apenas uma categoria de tamanho. Pariana campestris apresentou menos de $1 \%$ dos indivíduos na CT1, enquanto que 75,39\% estavam na CT3. Esta dominância também foi observada para Anthurium sinuatum com $86,70 \%$ dos indivíduos apenas na CT2. Para Oliveira e Amaral (2005), as espécies que concentram muitos indivíduos em uma categoria tendem a ser mais representativas nos parâmetros verticais, sendo importantes na estrutura desse ambiente, exercendo principalmente a função de cobertura do solo.

As ervas tiveram a maior porcentagem de indivíduos e regeneração natural relativa, correspondendo a $45,97 \%$ e $37,83 \%$ do total, respectivamente (Figuras $1 \mathrm{~A}$ e $1 \mathrm{~B}$ ). Enquanto que a forma de vida árvore foi predominante em número de espécies $(45,57 \%$ do total), seguida por erva $(17,72 \%)$, liana $(17,72 \%)$, arbusto $(8,86 \%)$, hemiepífita $(5,06 \%)$, palmeira $(3,80 \%)$ e epífita $(1,27 \%)$ (Figura 1C).

A maior porcentagem de ervas em número de indivíduos e regeneração natural relativa foi contrastante a outros estudos realizados no estuário amazônico, que no geral indicaram a maior concentração de indivíduos da forma de vida árvore (GAMA et al., 2003; MAUÉS et al., 2011). Lau e Jardim (2014), de forma semelhante ao presente estudo, observaram a maior presença de indivíduos herbáceos no banco de semente do solo da APA Combu e concluíram que as atividades antrópicas voltadas ao manejo da palmeira-açaí (Euterpe oleracea Mart.) bem como 
as inundações diárias e a alta intensidade luminosa no interior da floresta sejam possíveis fatores determinantes para a expressiva representatividade das ervas.

Figura 1 - Porcentagem de indivíduos (A), regeneração natural Rn\% (B) e espécies $(C)$ por forma de vida no estrato inferior de uma floresta ombrófila densa aluvial, Área de Proteção Ambiental Ilha do Combu. Arb = Arbusto; Arv = Árvore; Epi = Epífita; Erv = Erva; Hem = Hemiepífita; Lia $=$ Liana; Pal $=$ Palmeira.

Figure 1 - Percentage of individuals (A), Rn\% (B) and Species (C) by form of life in lower stratum in an alluvial dense rain forest in the Environmental Protection Area of Combu. Arb= Shrub; Arv = Tree; Epi = Epiphyte; Erv = Herb; Hem = Hemiepiphyte; Lia = Liana $;$ Pal= Palm tree.

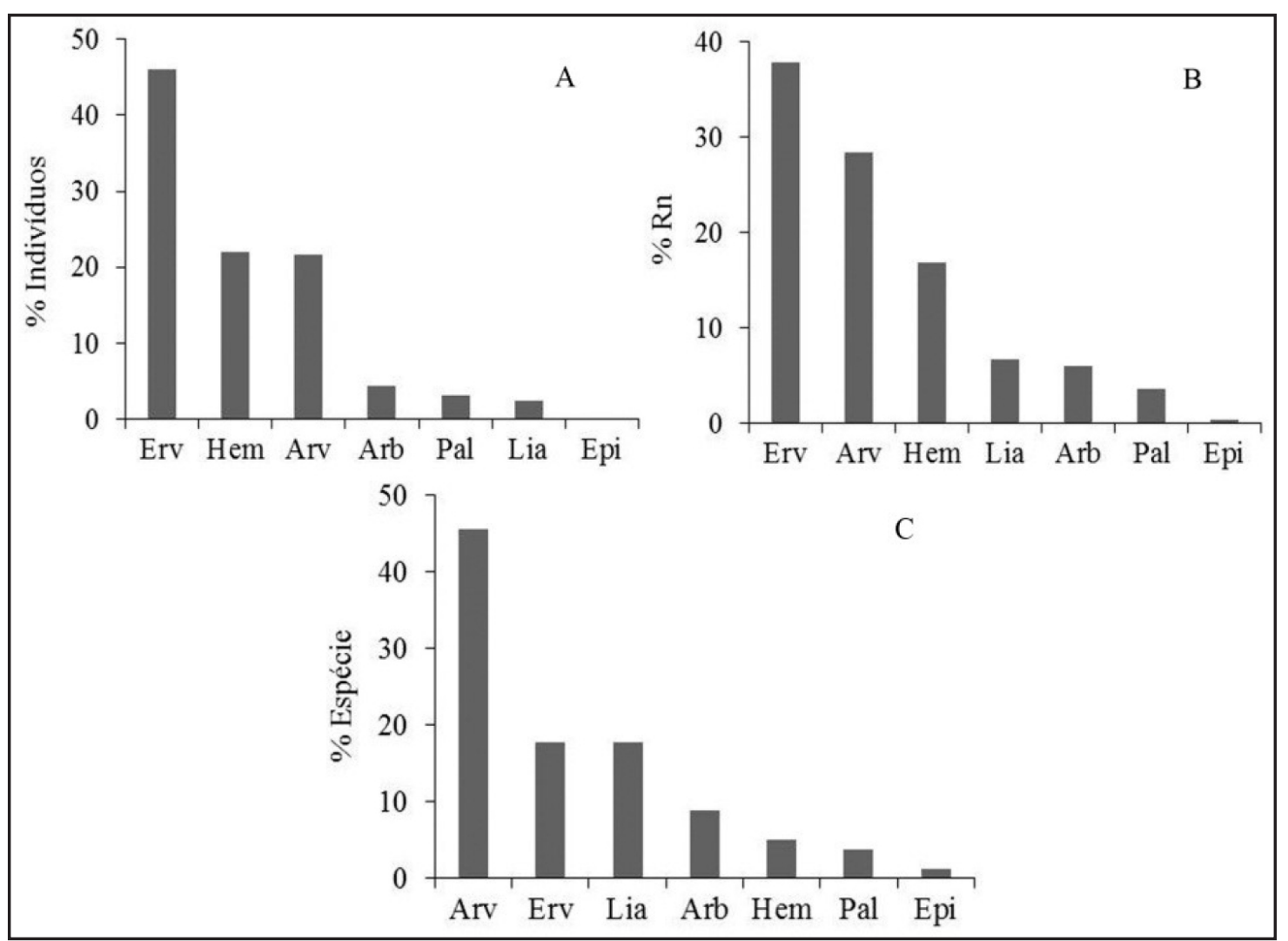

Fonte: Autores (2019)

A dominância de ervas nas florestas de várzea pode estar associada a diversas adaptações de algumas espécies pertencentes a esta forma de vida, como a resistência das sementes contra inundação; ciclos reprodutivos curtos; altas taxas de reprodução assexuada e em alguns casos o hábito de vida flutuante (JUNK; PIEDADE, 1993). Ervas são colonizadoras de ambientes com algum nível de perturbação, iniciando o processo de sucessão ecológica da floresta de várzea (CONSERVA; PIEDADE, 1998; PIEDADE; SCHOENGART; JUNK, 2005).

A predominância de árvores em porcentagem de espécie em relação às demais formas de vida foi similar ao relatado por Maués et al. (2011) em uma floresta ombrófila densa aluvial e por Jardim et al. (2013) em uma floresta de restinga inundável, sugerindo a influência da forma de vida árvore na estrutura da floresta de várzea.

As categorias de tamanho apresentaram 42 espécies em comum, 18 ocorreram somente em duas categorias, enquanto que 11 espécies ocorreram somente na CT2, 8 espécies na CT3 
e nenhuma espécie ocorreu exclusivamente em CT1. A Equabilidade e o Índice de ShannonWienner não diferiram entre as categorias. Mas houve diferença significativa entre a abundância e riqueza, sendo a média de indivíduos e espécies de CT1 menor em comparação com CT2 e CT3 (Tabela 2).

A ocorrência de algumas espécies em apenas uma categoria (CT2 ou CT3) pode ser resultante da ocupação agressiva das ervas, estas formam densos agrupamentos que podem, em longo prazo, levar à supressão de árvores no estrato inferior (GUILHERME; RESSEL, 2001), principalmente das espécies que necessitam de presença de luz para se desenvolver. Além disso, o manejo de Euterpe oleracea na floresta de várzea leva ao desaparecimento de espécies de árvores (FREITAS et al., 2015), possivelmente porque a limpeza e o raleamento da vegetação no entorno para favorecer o desenvolvimento do açaizeiro inibe a presença da fauna dispersora. A zoocoria, por exemplo, é importante na manutenção e na frequência das espécies de estágios sucessionais tardios na floresta (FRANCO et al., 2014).

A menor abundância e riqueza de CT1 em comparação com as demais categorias sugere um desequilíbrio na estrutura florestal, possivelmente por problemas relacionados aos mecanismos de regeneração das espécies, como a chuva de sementes e o banco de sementes. Estes mecanismos mantêm a dinâmica do processo de regeneração das espécies e determinam a estrutura da floresta (AVILA et al., 2013). As comunidades podem ser limitadas pelo baixo suprimento de sementes, ocasionado pela escassez de indivíduos produtores de diásporos (CAMPOS et al., 2009).

Tabela 2 - Média e desvio padrão da Abundância, Riqueza, Equabilidade e Índice de Shannon encontrados nas categorias de tamanho $(C T 1: 15 \mathrm{~cm} \geq A T$; CT2: $15,1 \mathrm{~cm} \leq A T \leq 30 \mathrm{~cm}$; CT3: $30,1 \mathrm{~cm} \leq \mathrm{AT} \leq 1 \mathrm{~m})$. Valores de $\mathrm{F}$ e p se referem ao teste estatístico Anova One-Way.

Table 2 - Average values and standard deviation of Abundance, Richness, Equability and Shannon index founded in the three size categories (CT1: $15 \mathrm{~cm} \geq A T$; CT2: $15,1 \mathrm{~cm} \leq \mathrm{AT} \leq 30$ $\mathrm{cm}$; CT3: $30,1 \mathrm{~cm} \leq \mathrm{AT} \leq 1 \mathrm{~m})$. $\mathrm{F}$ and $\mathrm{p}$ values refer to Anova One-Way statistic test.

\begin{tabular}{lcccc}
\hline $\begin{array}{c}\text { Categoria de } \\
\text { tamanho }\end{array}$ & Abundância & Riqueza & Equabilidade & Shannon \\
\hline CT1 & $339,25 \pm 52,23 \mathrm{a}$ & $24,75 \pm 2,75 \mathrm{a}$ & $0,67 \pm 0,08 \mathrm{a}$ & $2,13 \pm 0,22^{-\mathrm{a}}$ \\
CT2 & $3.486 \pm 1.683,19 \mathrm{~b}$ & $42,25 \pm 3,40 \mathrm{~b}$ & $0,61 \pm 0,03 \mathrm{a}$ & $2,28^{ \pm} 0,14^{\mathrm{a}}$ \\
CT3 & $2.896 \pm 1.017,07 \mathrm{~b}$ & $43 \pm 1,83 \mathrm{~b}$ & $0,56 \pm 0,15 \mathrm{a}$ & $2,08 \pm 0,56^{\mathrm{a}}$ \\
\hline & $\mathrm{F}(2,9)=8,675$ & $\mathrm{~F}(2,9)=56,88$ & $\mathrm{~F}(2,9)=1,219$ & $\mathrm{~F}(2,9)=0,324$ \\
& $\mathrm{p}=0,008$ & $\mathrm{p}=0,0001$ & $\mathrm{p}=0,343$ & $\mathrm{p}=0,731$ \\
\hline
\end{tabular}

Em que: Médias seguidas pela mesma letra na coluna não diferem entre si pelo teste de Tukey $(\mathrm{p}<0,05)$.

\section{Conclusão}

A diversidade do estrato inferior foi baixa, mas está dentro dos padrões comumente observados na floresta ombrófila densa aluvial. A dominância de ervas em porcentagem de indivíduos e regeneração natural relativa e a menor abundância e riqueza em CT1, indicam que as atividades antrópicas, como o manejo da palmeira-açaí estão afetando o estrato inferior das áreas que não contemplam esta atividade. Possivelmente, a limpeza e o desbaste para favorecer o desenvolvimento da palmeira estão inibindo os mecanismos de regeneração, afugentando a fauna dispersora e abrindo clareiras que favorecem a maior disseminação de ervas. 


\section{Agradecimentos}

Ao Conselho Nacional de Desenvolvimento Científico e Tecnológico (CNPq) pela concessão da bolsa de mestrado a primeira autora e pelo apoio ao projeto de Bolsa de Produtividade "Palmeiras da Amazônia Oriental como indicadoras de conservação ambiental e qualidade de vida” (Processo 305667/2013-0). Ao técnico Carlos Alberto Silva pela identificação das plantas.

\section{Referências}

ALMEIDA, A. F.; JARDIM, M. A. G. Florística e estrutura da comunidade arbórea de uma floresta de várzea na Ilha de Sororoca, Ananindeua, Pará, Brasil. Scientia Forestalis, Piracicaba, v. 39, n. 90, p. 191-198, jun. 2011.

ALMEIDA, S. S.; AMARAL, D. D.; SILVA, A. S. L. Análise florística e estrutura de florestas de várzea no estuário amazônico. Acta Amazonica, Manaus, v. 34, n. 4, p. 513-524, out./dez. 2004.

AMARAL, D. D. et al. The status of conservation of urban forests in eastern Amazonia. Brazilian Journal of Biology, São Carlos, v. 72, n. 2, p. 257-265, maio 2012.

ANGIOSPERM PHYLOGENY GROUP III. An update of the Angiosperm Phylogeny Group classification for the orders and families of flowering plants: APG III. Botanical Journal of the Linnean Society, London, v. 161, n. 2, p. 105-121, out. 2009.

APARICIO, P. S. et al. Níveis de regeneração natural em floresta de terra firme no Amapá Brasil. Revista Árvore, Viçosa, MG, v. 38, n. 4, p. 699-710, jul./ago. 2014.

AVILA, A. L. et al. Mecanismos de regeneração natural em remanescente de Floresta Ombrófila Mista, RS, Brasil. Cerne, Lavras, v. 19, n. 4, p. 621-628, out./dez. 2013.

BATISTA, F. J. et al. Comparação florística e estrutural de duas florestas de várzea no estuário amazônico, Pará, Brasil. Revista Árvore, Viçosa, MG, v. 35, n. 2, p. 289-298, mar./abr. 2011.

BATISTA, F. J.; JARDIM, M. A. G. Florística e estrutura da regeneração natural arbórea de uma floresta de várzea na Reserva Extrativista Chocoaré-Mato Grosso, Pará, Brasil. Biota Amazônia, Macapá, v. 3, n. 3, p. 139-145, 2013.

CAMPOS, E. P. et al. Chuva de sementes em Floresta Estacional Semidecidual em Viçosa, MG, Brasil. Acta Botanica Brasilica, São Paulo, v. 23, n. 2, p. 451-458, abr./ jun. 2009.

CARIM, M. J. V.; JARDIM, M. A. G.; MEDEIROS, T. D. S. Composição florística e estrutura de floresta de várzea no município de Mazagão, Estado do Amapá, Brasil. Scientia Forestalis, Piracicaba, v. 36, n. 79, p. 191-201, set. 2008.

CARVALHO, J. et al. Relações entre a distribuição das espécies de diferentes estratos e as características do solo de uma floresta aluvial no Estado do Paraná, Brasil. Acta Botanica Brasilica, São Paulo, v. 23, n. 1, p. 1-9, jan./mar. 2009.

COELHO, R. F. R. et al. Análise florística e estrutural de uma floresta em diferentes estágios sucessionais no município de Castanhal, Pará. Acta Amazonica, Manaus, v. 33, n. 4, p. 563-582, dez. 2003.

CONSERVA, A.; PIEDADE, M. T. F. Influence of flood-pulse and land-use on the composition of herbaceous species on a floodplain in Central Amazonia. Verhandlungen Internationale Vereinigung für Theoretische und Angewandte Limnologie, Stuttgart, v. 26, p. 994-995, mar. 1998.

FINOL, U. H. Nuevos parámetros a considerarse em el análisis estructural de las selvas vírgenes tropicales. Revista Florestal Venezolana, [s. 1.], v. 14, n. 21, p. 29-42, 1971. 
FOX, J. et al. Companion to Applied Regression: Package 'car'. Disponível em: $<$ http://cran.rproject.org/>. Acesso em: 08 ago. 2015.

FRANCO, B. K. S. et al. Estrato de regeneração natural de um trecho de Floresta Estacional Semidecidual, Viçosa, MG. Revista Árvore, Viçosa, MG, v. 38, n. 1, p. 31-40, jan./fev. 2014.

FREITAS, M. A. B. et al. Floristic impoverishment of Amazonian floodplain forests managed for açaí fruit production. Forest Ecology and Management, Amsterdam, v. 351, n. 1, p. 20-27, sep. 2015.

GAMA, J. R. V.; BOTELHO, S. A.; BENTES-GAMA, M. M. Composição florística e estrutura da regeneração natural de floresta secundária de várzea baixa no estuário amazônico. Revista Árvore, Viçosa, MG, v. 26, n. 5, p. 559-566, set./out. 2002.

GAMA, J. R. V. et al. Estrutura e potencial futuro de utilização da regeneração natural de floresta de várzea alta no município de Afuá, Estado do Pará. Ciência Florestal, Santa Maria, v. 13, n. 2, p. 71-82, dez. 2003.

GUILHERME, F. A. G.; RESSEL, K. Biologia floral e sistema de reprodução de Merostachys riedeliana (Poaceae: Bambusoideae). Revista Brasileira de Botânica, São Paulo, v. 24, n. 2, p. 205-211, jun. 2001.

IBGE. Manual técnico da vegetação brasileira. Rio de Janeiro: Fundação Instituto Brasileiro de Geografia e Estatística, 2012. 271 p.

JARDIM, D. G. et al. Regeneração natural em formações florestais de uma Unidade de Conservação, Maracanã, Pará, Brasil. Biota Amazônia, Macapá, v. 3, n. 2, p. 79-87, 2013.

JARDIM, M. A. G.; VIEIRA, I. C. G. Composição florística e estrutura de uma floresta de várzea do estuário amazônico, Ilha do Combu, Estado do Pará, Brasil. Boletim do Museu Paraense Emílio Goeldi, Ciências Naturais, Belém, v. 17, n. 2, p. 333-354, 2001.

JUNK, W. J.; PIEDADE, M. T. F. Herbaceous plants of the Amazon floodplain near Manaus: Species diversity and adaptations to the flood pulse. Amazoniana, Kiel, v. 12, n. 3/4, p. 467-484, dez. 1993.

LAU, A. V.; JARDIM, M. A. G. Composição e densidade do banco de sementes em uma floresta de várzea, Ilha do Combu, Belém-PA, Brasil. Biota Amazônia, Macapá, v. 4, n. 3, p. 5-14, 2014.

LAU, A. V.; JARDIM, M. A. G. Florística e estrutura da comunidade arbórea em uma floresta de várzea na Área de Proteção Ambiental, Ilha do Combu, Belém, Pará. Biota Amazônia, Macapá, v. 3, n. 2, p. 88-93, 2013.

MAGURRAN, A. E. Ecological diversity and its measurement. New Jersey: Princeton University Press, 1988. 179 p.

MAUÉS, B. A. R. Composição florística e estrutura do estrato inferior de floresta de várzea estuarina na Área de Proteção Ambiental Ilha do Combu, Belém-PA, Brasil. 2009. $60 \mathrm{f}$. Dissertação (Mestrado em Ciências Biológicas) - Universidade Federal Rural da Amazônia, Belém, 2009.

MAUÉS, B. A. R. et al. Composição florística e estrutura do estrato inferior da floresta de várzea na área de proteção ambiental Ilha do Combu, município de Belém, Estado do Pará. Revista Árvore, Viçosa, MG, v. 35, n. 3, p. 669-677, maio/jun. 2011.

MARINHO, T. A. S. et al. Distribuição e crescimento de Garcinia brasiliensis Mart. e Hevea spruceana (Benth.) Müll. Arg. em uma floresta inundável em Manaus, Amazonas. Ciência Florestal, Santa Maria, v. 23, n. 1, p. 223-232, jan./ mar. 2013.

MENDES, F. S. et al. Dinâmica da estrutura da vegetação do sub-bosque sob influência da exploração em uma floresta de terra firme no município de Moju-PA. Ciência Florestal, Santa Maria, v. 23, n. 2, p. 377-389, abr./ jun. 2013. 
MISSOURI BOTANICAL GARDEN. [Website]. Disponível em: $<$ http:/www.tropicos.org $>$ Acesso em: 01 ago. 2015.

OLIVEIRA, A. N.; AMARAL, I. L. Aspectos florísticos, fitossociológicos e ecológicos de um sub-bosque de terra firme na Amazônia Central, Amazonas, Brasil. Acta Amazonica, Manaus, v. 35, n. 1, p. 1-16, 2005.

PIEDADE, M. T. F.; SCHOENGART, J.; JUNK, W. J. O manejo sustentável das áreas alagáveis da Amazônia Central e as comunidades de herbáceas aquáticas. Uakari, Manaus, v. 1, n. 1, p. 29-38, nov. 2005.

PIELOU, E. C. Mathematical Ecology. New York: Wiley, 1977. 165 p.

QUARESMA, A. C.; JARDIM, M. A. G. O manejo do açaizeiro, Euterpe oleracea Mart., influencia a diversidade de bromélias epifítas em floresta de várzea? Biota Amazônia, Macapá, v. 3, n. 2, p. 94-100, jul./set. 2013.

R CORE TEAM. R: a language and environment for statistical computing. Viena: R Foundation for Statistical Computing, 2014. Disponível em: <http://www.r-project.org/>. Acesso em: 08 ago. 2015.

RABELO, F. G. et al. Regeneração natural de florestas estuarinas na região do Rio AmazonasAmapá-Brasil. Revista de Ciências Agrárias, Belém, n. 34, p. 129-137, jul./dez. 2000.

ROCHA, A. E. S.; LINS, A. L. F. A. Checklist das Poaceae de áreas inundáveis e inundadas do nordeste do estado do Pará. Acta Amazonica, Manaus, v. 39, n. 4, p. 763-772, 2009.

SANTOS, G. C. et al. Fitossociologia e práticas de manejo tradicional em uma floresta de várzea em Santa Bárbara do Pará, Estado do Pará, Brasil. Revista Ciências Agrárias, Belém, v. 57, n. 2, p. 138-145, abr./jun. 2014.

SANTOS, G. C.; JARDIM, M. A. G. Florística e estrutura do estrato arbóreo de uma floresta de várzea no município de Santa Bárbara do Pará, Estado do Pará, Brasil. Acta Amazonica, Manaus, v. 36, n. 4, p. 437-446, out./dez. 2006.

SODERSTROM, T. R.; CALDERÓN, C. E. Insect pollination in tropical rain forest grasses. Biotropica, [s. 1.], v. 3, n. 1, p. 1-16, jun. 1971.

SMITH, A. R. et al. A classification for extant ferns. Taxon, [s. 1.], v. 55, n. 3, p. 705-731, aug. 2006.

VELOSO, H. P.; RANGEL FILHO, A. L. R.; LIMA, J. C. A. Classificação da vegetação brasileira adaptada a um sistema universal. Rio de Janeiro: IBGE, 1991. 124 p.

VIANA, A. C. N.; JARDIM, F. C. S. Natural regeneration dynamics of Couratari guianensis in a tropical forest selectively logged in Moju, state of Para, Brazil. Revista de Ciências Agrárias, Belém, v. 56, n. 2, p. 112-119, abr./jun. 2013.

WITTMANN, F.; JUNK, W. J. Sapling communities in Amazonian white-water forests. Journal of Biogeography, Oxford, v. 30, n. 10, p. 1533-1544, oct. 2003. 